\title{
JH2R: Joint Homography Estimation for Highlight Removal
}

\section{Sungmin Eum}

smeum@umiacs.umd.edu

Hyungtae Lee

htlee@umiacs.umd.edu

David Doermann

doermann@umiacs.umd.edu
University of Maryland Institute of Advanced Computer Studies (UMIACS)

University of Maryland College Park

College Park, MD, USA

Imagine being in an art museum where there are paintings or pictures held inside glass-frames for protection. There are pieces which you wish to capture using a camera, but you experience difficulties avoiding highlights which are generated by indoor lighting reflected off the glossy surfaces. Similar problems occur when capturing contents off of whiteboards, documents printed on glossy surfaces, objects such as books or CDs with plastic covers.

In this work, we address the problem of removing unwanted highlight regions in images generated by reflections of light sources on glossy surfaces. Although there have been efforts made to synthetically fill in the missing regions using the neighboring patterns by applying methods like inpainting [3, 4], it is impossible to recover the missing information in completely saturated regions. Therefore, we need to use multiple images where corresponding regions are not covered by the saturated highlights. Unlike other methods, our method uses the relationship between the highlight regions resulting in more robust removal of saturated highlights.

Our method - Overview Our method was motivated by a widely acknowledged physical phenomenon referred to as the 'motion parallax'. Without loss of generality, we can similarly view the relationship between the desired content (e.g., a painting) and the highlights. Since the highlights caused by the light source are the result of the reflection on the glossy surface before they reach the camera, the light source can be modeled to virtually exist on the other side of the content. Note that, the distance from the light source is always larger than the distance from the content $(D>d$, in Figure 1$)$.

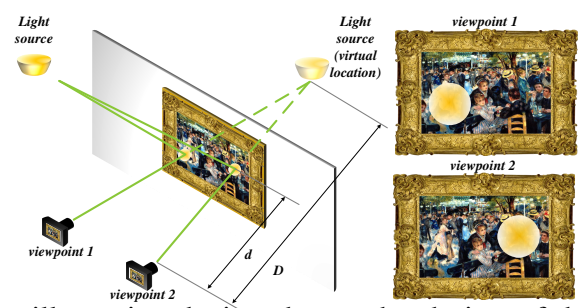

Figure 1: The illustration depicts the overhead view of the camera, the desired content, and the light source.

In order to distinguish the movements of the highlights, we need at least two images captured from different views. We then detect where the highlights are by searching for the two separate homography matrices: one for the content $\left(\mathbf{H}_{C}\right)$ and the other for the highlights $\left(\mathbf{H}_{H}\right)$. We exploit the fact that the homography $\left(\mathbf{H}_{C}\right)$ which can properly overlay the desired contents in the two images will generate an erroneous overlap between the corresponding highlight regions. Similarly, the desired contents will display incorrect overlap when $\mathbf{H}_{H}$ is employed. This is shown in the second step of Figure 2(b).

Unlike the intrinsic layer separation problems, removing the saturated highlights from the images requires another image which can provide the corresponding non-highlight pixels. To perform such "pixel-transfer", it is necessary to have the pixel-level detection results of the highlights. In

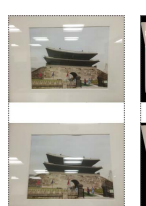

(a) (b)

Figure 2: Schematic overview of our method (a) Input images (b) Joint homography estimation (c) Feature-level labeling (d) Pixel-level labeling (e) Final results our approach, we first detect the highlight regions at the feature level by jointly estimating the two homographies using the proposed Joint Homography Estimation for Highlight Removal (JH2R) algorithm. However, the feature-level detection of the highlight regions is insufficient to properly eliminate the highlights. Thus, $\mathbf{H}_{H}$ is used to estimate the highlight regions at the pixel-level. Finally, we remove the highlights in both of the images by transferring the corresponding pixels from the complementary image using Poisson blending [4]. Figure 2 shows the schematic overview of our method. Details of the algorithm are explained in the original manuscript.

Comparison with state-of-the-art We have compared our method with four state-of-the-art algorithms $[1,2,3,5]$. They are chosen to represent three different approaches to solve the given problem : 1) highlight removal, 2) single image-based reflection removal, and 3) multiple imagebased reflection removal.

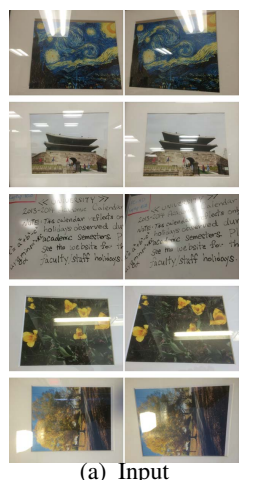

(a) Input

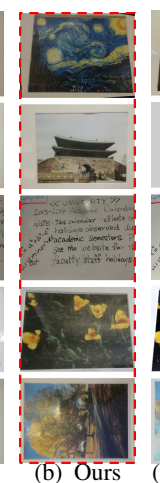

(b) Oūrs
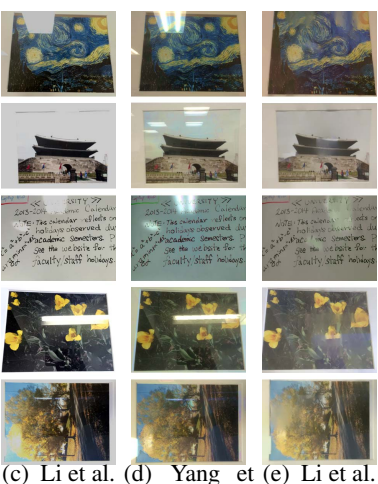

Figure 3: Five examples of highlight removal results using (b) our method compared with those produced by (c) Li et al. [3], (d) Yang et al. [5], (e) Li et al. [2], (f) Guo et al. [1]

Figure 3 shows five sample results of real world images. As can be observed in Figure 3(c) and 3(d), both [3] and [5] are incapable of removing the highlights due to the lack of information within the regions. Multiple-image based approaches (Figure 3(e) and 3(f)) by [1, 2] produce results where the highlights are only partially removed. Figure 3(e). Our method, unlike others, specifically uses the relationship between the "highlight" regions resulting in more precise detection and removal. One may observe from Figure 3 that our method can also handle dim highlights as there still exist geometrical distinction between desired contents and dim highlights in terms of homography. In overall, our method produces the most visually pleasing results.

[1] X. Guo, X. Cao, and Y. Ma. Robust separation of reflection from multiple images. In Computer Vision and Pattern Recognition (CVPR), IEEE Computer Society Conference on., pages 2195-2202, 2014.

[2] Y. Li and M.S. Brown. Exploiting reflection change for automatic reflection removal. In Computer Vision (ICCV), IEEE International Conference on, pages 2432-2439, 2013.

[3] Y. Li and M.S. Brown. Single image separation using relative smoothness. In Computer Vision and Pattern Recognition (CVPR), IEEE Computer Society Conference on., pages 2752-2759, 2014.

[4] P. Pérez, M. Gangnet, and A. Blake. Poisson image editing. ACM Transactions on Graphics, 22(3):313-318, Jul 2003.

[5] Q. Yang, J. Tang, and N. Ahuja. Efficient and robust specular highlight removal. Pattern Analysis and Machine Intelligence, IEEE Transactions on, 1(1):1, 12014. 